\title{
Papel del diálogo como estrategia oral en la comprensión de textos expositivos en ambientes de aprendizaje b-learning
}

\author{
Sandra Patricia Quitián Bernal*
}

Fecha de recibido: 18 de julio de 2011

Fecha de aprobado: 23 de noviembre de 2011

\section{Resumen}

Se presentan resultados de una investigación de corte cuasiexperimental fundamentada en reflexiones teóricas sobre la relación entre la conversación oral y la comprensión de textos expositivos. La problemática concierne a la relación entre la formación de lectores competentes en materiales de lectura de tipo expositivo y su relación con las acciones didácticas que lideran los docentes desde cada disciplina, antes, durante y después del proceso lector. En la medida en que los resultados de la investigación demuestran que este proceso posibilita el desarrollo y la exteriorización de dominios cognitivos y discursivos relacionados con la comprensión y, por tanto, requiere de mecanismos de seguimiento y control, mediados por el maestro desde el uso de estrategias orales, este artículo describe la propuesta de estrategias particulares que favorecen dicho proceso. Por ello, se propone el aprendizaje en modalidad $b$-learning como una alternativa importante para apoyar la comprensión lectora de textos expositivos desde la vinculación de diferentes recursos de aprendizaje.

Palabras Clave: oralidad, comprensión, estrategias, texto expositivo, ambiente b-learning.

\section{Abstract}

This article shows the results of a quasi-experimental investigation, based on theoretical reflections on the relationship between oral conversation and expository text comprehension. The problems concerning the relationship between the formation of competent readers in expository texts and their relation with the teaching activities that lead teachers from each discipline, before, during and after the reading process. To the extent that the results of the investigation show that this process enables the development and the externalization of cognitive and discursive domains related to understanding and, therefore, requires monitoring and control mechanisms, mediated by the teacher from using oral strategies. This paper describes the proposal of particular strategies, which favor the process. Therefore, we propose the learning environment b-learning as an important alternative to support reading comprehension of expository text from the linking of different learning resources.

Key words: orality, understanding, strategies, expository text, b-learning environment

\footnotetext{
Normal Superior Distrital María Montessori. Correo: squitian226@gmail.com
} 


\section{Introducción}

La lectura en el espacio académico está normalmente asociada con materiales de orden expositivo. El dominio exigido para este tipo de lectura requiere de un proceso de formación y trabajo escolar teóricopráctico, orientado por los docentes, pues los modos de leer y aproximarse de forma efectiva a los textos no son inherentes al ser humano, es necesario aprenderlos. Si bien los textos expositivos son comúnmente los más usados en la actividad académica, esto no significa que estudiantes y docentes cuenten siempre con un dominio suficiente para abordar su tratamiento en diferentes contextos de la vida escolar.

No es de extrañar que en repetidas ocasiones la exigencia de comprensión de algunos textos trabajados en el aula supere la capacidad comprensiva con que cuentan los escolares. Esta circunstancia podría evitarse si el docente conoce con antelación las exigencias de comprensión que demandan los textos que utiliza en el desarrollo de las clases y logra anticiparse a las dificultades de sus estudiantes. De ahí que la formación de lectores competentes en materiales de lectura de tipo expositivo dependa fundamentalmente, entre otros aspectos, de la clase de acciones didácticas que lideren los docentes desde cada disciplina, en el antes, el durante y el después del proceso lector.

El papel de la lectura es clave para acceder al dominio de diferentes campos del conocimiento. Sin embargo, no basta con "leer" para "comprender". El aprendizaje es posible cuando se puede interpretar la información nueva en términos de conocimientos y conceptos adquiridos previamente; son estos los que le permiten al lector entender e interpretar la información y elaborar una representación mental coherente de lo que el texto presenta (López G y E. Arciniegas, 2004). Este proceso de reconstrucción requiere en muchos casos de la interacción y mediación del lector con otros, bien sea el docente o los pares académicos.
La exteriorización del proceso de comprensión se hace desde el uso del potencial lingüístico del sujeto que lee. La lengua oral, como materialización del lenguaje y producto de procesos cognitivos, afectivos y sociales, que van más allá de la acción cotidiana de hablar, se instala en el marco de las experiencias de aprendizaje, y particularmente en el escenario del aula de clase, como el principal medio de interacción y mediación entre el texto, el lector y el autor. Situarnos en la relación lengua oralcomprensión pone de manifiesto que esta última se explicite como un proceso construido desde la interacción del sujeto con diferentes elementos internos y externos, donde necesariamente tiene presencia la oralidad. El uso de estrategias orales para favorecer la comprensión de los textos expositivos se hace cada vez más necesario en el aula y desde todas las materias de contenido.

En consecuencia, la tecnología ofrece un recurso importante mediante la implementación de ambientes de aprendizaje b-learning (blended learning). En esta modalidad, docentes y estudiantes cuentan con dos escenarios de interacción diferentes, pero complementarios: el espacio presencial (aula de clase) y el espacio virtual (aula virtual), los cuales son mediados por interacciones orales y escritas.

\section{Referentes conceptuales de la investigación}

\section{Lectura, oralidad y aprendizaje}

Varios autores han coincidido en definir el acto de leer como un proceso de construcción de significado que se da a partir de la relación que el lector establece con el texto. Ese significado no está en el texto, pero tampoco en el lector. Es producto de la interacción que se establece entre ellos, de la cual depende en buena medida la comprensión. Desde los planteamientos de Solé (2001), la lectura puede referirse como un "proceso de construcción que in- 
volucra tanto al texto y a los conocimientos previos del lector, como a los objetivos que lo mueven a leer".

Existen estrechas relaciones entre la lengua oral y los procesos asociados a la comprensión, ya que los usos de producción verbal de los sujetos suelen estar determinados, además de la intención, también por el nivel de entendimiento de la persona sobre la situación o la información que posibilita este producto oral. La oralidad, entendida como "modalidad de realización del lenguaje humano, que involucra los procesos discursivos de hablar y escuchar" (Jaimes, 2005) es en sí misma una actividad de pensamiento determinada por las condiciones situacionales particulares en las que se produce. La condición dialógica del lenguaje humano tiene su punto de partida y de encuentro en la oralidad, pues toda interacción mediante la palabra hablada se dirige a otro en busca de respuesta, en busca de un interlocutor con el cual tejer una relación social y jerárquica (Vigotsky, 1989). Esta naturaleza dialógica no es ajena a los procesos de desarrollo del lenguaje en el espacio escolar.

Particularmente, en relación con la lectura, interesa reflexionar sobre los modos particulares de aprender y de enseñar estrategias que favorezcan la comprensión, desde la vinculación de la oralidad como proceso mediador entre el lector, el autor y el texto. Rosales (1997, citado por Núñez, 2000) señala que entre mayores sean las competencias de comprensión y expresión oral de los estudiantes al ingresar a la escuela, mayores serán sus posibilidades de aprender a leer y a escribir con éxito.

La comprensión es un proceso interactivo y dinámico, puesto que el lector pone en diálogo los objetivos y propósitos que lo guían, la información que el escrito le proporciona y los conocimientos que posee para construir el significado del texto. Los conocimientos que se organizan y almacenan en la memoria y que guían al lector en los procesos de comprensión e interpretación del texto se conocen como esquemas.

Entonces, existe relación cercana entre aprendizaje, oralidad y lectura. El uso de estrategias orales en el aula de clase, favorece la presencia de interacciones verbales, orientadas a la activación y uso eficiente de esquemas cognitivos y conocimientos previos del lector. El aprendizaje pasa, en consecuencia, por el procesamiento, la comprensión y la retención adecuada de los contenidos expresados en el texto. La lectura se constituye así en una actividad mental de alto nivel en la que los esquemas cognitivos son fundamentales para la comprensión y el aprendizaje (Solé, 1992).

\section{La comprensión de textos expositivos desde el modelo de enseñanza recíproca}

La comprensión, referida como uno de los procesos cognitivos más importantes, demanda cosas que van más allá de la "habilidad o destreza para entender lo que se lee". Para el desarrollo de esta investigación se optó por trabajar con el modelo de enseñanza de la comprensión propuesto por Palincsar y Brown (1984), al que llamaron "enseñanza recíproca”. Estas psicólogas elaboraron un paquete instruccional compuesto por cuatro actividades cognitivas relacionadas con la comprensión: predicción, clarificación, resumen, autopregunta, las cuales se aplicaban en contextos educativos tradicionales.

Las autoras de este modelo de enseñanza señalan su inspiración en la teoría de aprendizaje de Vigotsky (1978), el cual parte de que el aprendizaje supone la interiorización de actividades originalmente adquiridas y practicadas en situaciones de cooperación social. Es decir, los estudiantes aprenden participando en actividades grupales en las cuales son expuestos a modelos (el maestro) que difieren en su nivel de ejecución experta. Poco a poco, los miembros más capacitados del grupo (estudiantes) empie- 
zan a ser modelo y enseñan gradualmente a los más inexpertos, proporcionándoles asistencia, hasta que estos están en disposición de ejecutar la tarea por sí mismos.

El trabajo de modelamiento en el que se sustenta la "enseñanza recíproca" exige del maestro no solo el desarrollo instruccional, sino además un adecuado uso del diálogo, de la conversación. La conversación, como estrategia oral, la define Gutiérrez (1997) como un conjunto de actos comunicativos en los que dos o más sujetos hablantes intercambian mensajes orales, alternando sus funciones de emisor y receptor, de forma libre y espontánea, en unas coordenadas de simultaneidad espacial y temporal. Para López (1996), la técnica de la conversación nos faculta para rechazar o confirmar nuestros puntos de vista gracias a las aportaciones que hacen los demás y a los nuevos planteamientos que se van dando al ir exponiendo las propias opiniones.

El trabajo de colaboración y construcción social que se promueve desde el ambiente de aprendizaje $b$ learning es relevante. Por medio del trabajo en pequeños equipos, cuyo nivel de ejecución es mediado por el nivel de mayor o menor dominio de sus integrantes, el maestro permanentemente anima desde la interacción verbal y el acompañamiento continuo, para que los estudiantes más adelantados logren jalonar en sus demás compañeros procesos de aprendizaje. La función dialógica que propicia el maestro con sus estudiantes es clave para apropiarse de estrategias de comprensión.

Aunque el proceso de comprensión es uno solo, no todos los textos se leen de la misma forma debido a las características textuales y discursivas particulares. En este sentido, el desarrollo de este trabajo vincula de modo particular las lecturas de tipo expositivo. La palabra "exponer" sugiere la noción de explicar un tema sobre cualquier asunto, con el fin de que los destinatarios lo conozcan o lo comprendan
Tabla 1. Modelo focalizado

\begin{tabular}{|c|l|}
\hline \multirow{2}{u}{} & MODELO FOCALIZADO \\
-ESTRATEGIAS DE LECTURA- \\
\hline \multirow{2}{*}{$\begin{array}{l}\text { ESTRATEGIAS DE ACTIVACIÓN DE ESQUEMAS } \\
\text { MENTALES }\end{array}$}
\end{tabular}

Fuente: elaboración propia. 
Tabla 2. Estrategias de lectura: modelo panorámico

\begin{tabular}{|c|c|}
\hline & $\begin{array}{l}\text { MODELO PANORÁMICO } \\
\text {-ESTRATEGIAS DE LECTURA- }\end{array}$ \\
\hline \multirow[b]{2}{*}{ 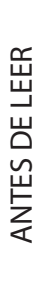 } & ESTRATEGIAS DE ACTIVACIÓN DE ESQUEMAS MENTALES \\
\hline & $\begin{array}{l}\text { Acciones y procedimientos que permiten disponer al lector para generar una relación interactiva y simultánea entre } \\
\text { sus esquemas cognitivos - todo el conocimiento previo-y la información nueva provista por el texto. } \\
\text { Establecer los propósitos de la lectura. } \\
\text { Activar el conocimiento previo (imágenes, títulos, subtítulos). } \\
\text { Formularse preguntas sobre aspectos específicos del texto. }\end{array}$ \\
\hline \multirow{4}{*}{ 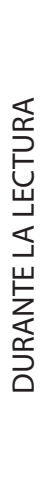 } & ESTRATEGIAS SELECTIVAS \\
\hline & $\begin{array}{l}\text { El fin de estas estrategias es realizar una lectura superficial del texto en la que se incluyen: } \\
\text { La lectura rápida, la lectura para atender aspectos de la información o para identificar el significado de palabras y } \\
\text { obtener un panorama general del contenido. }\end{array}$ \\
\hline & ESTRATEGIAS DE ORGANIZACIÓN \\
\hline & $\begin{array}{l}\text { El lector puede restructurar de forma distinta el texto a fin de hacerlo más significativo y comprensible. Por ejemplo: } \\
\text { Reorganizarlo a partir de determinados criterios, como importancia jerárquica de los acontecimientos o situaciones. } \\
\text { Identificación de la estructura expositiva seleccionando los elementos claves desde la misma. } \\
\text { Ordenamiento de información según fecha, categoría, secuencia, etc. }\end{array}$ \\
\hline \multirow{4}{*}{ 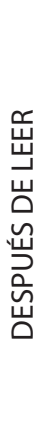 } & ESTRATEGIAS DE ELABORACIÓN \\
\hline & $\begin{array}{l}\text { Permiten integrar la información del texto con los conocimientos previos del lector a fin de comprender con más } \\
\text { profundidad el significado. } \\
\text { Formular conclusiones de tipo teórico o práctico. } \\
\text { Generar imágenes y analogías del texto leído. }\end{array}$ \\
\hline & ESTRATEGIAS DE COMPROBACIÓN \\
\hline & $\begin{array}{l}\text { Procura constatar la cohesión y la coherencia, así como la consonancia del texto con el tipo de estrategia, en re- } \\
\text { lación con el sentido del texto. Corresponde a un nivel altamente elaborado y del que solo dan cuenta aquellos } \\
\text { lectores que han adquirido cierto nivel de autonomía. }\end{array}$ \\
\hline
\end{tabular}

Fuente: elaboración propia.

mejor (Richgels y otros, 1990). Así, pues, se puede definir la exposición como el tipo de texto o discurso cuyo objeto es transmitir información para darla a conocer, para hacerla entender.

La exposición es, sin duda, la forma más habitual de expresión de las ideas, conocimientos, tratados cien- tíficos y técnicos, libros didácticos, prospectos de medicamentos y todos aquellos textos cuya finalidad consista en informar sobre hechos, conceptos o desarrollo de conocimientos. Los textos o discursos expositivos pueden adoptar diferentes estructuras. Anderson y Ambuster (1984) proponen seis estructuras textuales en las que se pueden organizar los

1 Denominación hecha para hacer referencia a la lectura de textos expositivos en el espacio escolar, en escuelas americanas. 
textos expositivos, cuyo uso se ha convertido en una necesidad recurrente en todos los niveles de escolaridad, pues a partir de la lectura se pretende que los estudiantes adquieran nuevos conocimientos.

Taboada y Guthrie (2006), a partir de investigaciones desarrolladas en los últimos años sobre los niveles de comprensión en materias de contenido ${ }^{1}$ en Estados Unidos, plantean dos variaciones dentro de la lectura de materias de contenido. Se trata de la lectura focalizada o de "nicho" (tabla 1) y la lectura panorámica o de "paisaje" (tabla 2).

La comprensión y claridad en el manejo de los vínculos y relaciones entre la lectura de "nicho", que es altamente contextualizada, y la lectura de "paisaje", que es ampliamente distribuida y global, son condiciones importantes en la formación de lectores competentes. Para efectos de esta investigación, la lectura focalizada y la panorámica se referencian como "modelos de lectura".

\section{Relaciones entre estrategias lectoras, modelos de lectura y estilos cognitivos}

Coll (1988) se refiere a las estrategias como un procedimiento -llamado también a menudo regla, técnica, método, destreza o habilidad-o conjunto de acciones ordenadas y finalizadas, es decir, dirigidas a la consecución de una meta. López y Arciniegas (2004), por su parte, definen la estrategia como "la secuencia de actividades intencionales y deliberadas en las cuales se involucra conscientemente el individuo para lograr las metas que se ha propuesto".

La enseñanza de las estrategias de lectura está orientada a la formación de lectores autónomos, capaces de enfrentar el procesamiento de cualquier tipo de texto. La autonomía en la lectura puede hacerse visible en la medida en que un lector es capaz de con- trolar y cuestionar la calidad de su propio proceso de lectura, además de las maneras como procede para construir los distintos significados del texto. Las estrategias lectoras ayudan, por tanto, a modelar las maneras de proceder con los textos, de acuerdo con los contenidos, las tareas lectoras y el tipo de complejidad que tengan estos.

En esta investigación, el uso de estrategias de lectura está directamente vinculado a los modelos Focalizado o Panorámico. Optar por un modelo panorámico o por un modelo focalizado depende en muchos casos no solo del tipo de lector que se sea, sino también del estilo cognitivo que caracteriza al sujeto frente al procesamiento de información. El estilo cognitivo se asume dentro de este trabajo como el conjunto de preferencias en el uso de las habilidades cognitivas y procedimentales que posee y opera un sujeto para interactuar y percibir el conocimiento. En este caso, se hace referencia al estilo cognitivo dependiente e independiente de campo.

De acuerdo con A. Lozano (2005), algunas de las características cercanas al estilo cognitivo dependiente de campo están relacionadas con la percepción global, mayor calidad en la memorización de información social, uso recurrente de instrucciones para realizar tareas, necesidad de permanente orientación e instrucciones de otros. Respecto a la personalidad, estos sujetos son sensibles a la crítica, no tienen muy definido el sentido de autonomía, les gusta trabajar con otros y, por ello, son fácilmente influenciables.

Por el contrario, el estilo cognitivo independiente de campo o analítico, como su nombre lo indica, maneja en mayor grado la percepción analítica. Se está más en función del trabajo personal que del trabajo social o de la interacción con otros. Quienes lo poseen, son capaces de resolver problemas y dar soluciones, sin necesitar las instrucciones de otros; 
poseen iniciativa y pueden utilizar sus propios criterios para desarrollar tareas. Las críticas no les afectan mucho, tienen un sentido definido de autonomía son estudiantes difícilmente influenciados.

Finalmente, la puesta en escena del entretejido entre estrategias, modelos de lectura y capacidades del sujeto lector requiere de un ambiente de aprendizaje que ofrezca alternativas diferentes a las tradicionales, pues estas no siempre responden a las expectativas intelectuales y sociales de todos los estudiantes.

\section{Metodología}

\section{Población}

El diseño de esta investigación de corte cuasi experimental permitió la organización de tres grupos, de acuerdo con el estilo cognitivo: Grupo 1: veintiséis estudiantes con estilo cognitivo dependiente; Grupo 2: veinticinco estudiantes con estilo cognitivo independiente; Grupo 3: veintiséis estudiantes con estilo cognitivo intermedio. Esta clasificación se dio gracias a la aplicación de la prueba de figuras enmascaradas (EFT). Los setenta y siete participantes que conformaron la muestra fueron jóvenes de grado undécimo cuyas edades oscilaban entre los quince y los diecisiete años, escogidos de forma intencional, pues hacían parte de dos grupos organizados por la Escuela Normal Superior Distrital María Montessori para el desarrollo del programa de Taller de Lectura y Escritura.

\section{Instrumentos}

Test de figuras enmascaradas: La prueba de figuras enmascaradas (EFT) es el instrumento usado para la determinación del estilo cognitivo en su dimensión de independencia-dependencia de campo. La prueba mide la velocidad de reestructuración perceptual, principal indicador de la tendencia hacia la independencia de campo en cada sujeto. Elaborada inicialmente por $\mathrm{H}$. Witkin y sus colaboradores en 1950, la versión grupal referenciada aquí fue desarrollada por Sawa (1966). La prueba consta de una serie de cinco ejercicios, cada uno de los cuales está constituido por una figura simple y diez figuras complejas diferentes. En cada ejercicio al sujeto se le pide que descubra y trace a lápiz el contorno de la figura simple en cada una de las diez figuras complejas. Esta tarea debe realizarse en un determinado tiempo.

EnCUeSTA METACOgNitiva: se aplicó una encuesta metacognitiva (López y Arciniegas, 2004), la cual busca determinar el conocimiento que los estudiantes manifiestan tener sobre los procesos que llevan a cabo, antes, durante y después de una lectura de estudio, para identificar el procedimiento que siguen y las estrategias que utilizan. La encuesta consta de 41 ítems distribuidos y con respuesta cerrada "Si" o "No" para cada uno. Esta encuesta se aplicó al inicio y al final del proceso de intervención.

REgisTROS DE OBSERVACIÓN DIRECTA: fueron empleados en distintos momentos del proceso y sobre descriptores puntuales que indagan por la actitud y disposición de los estudiantes respecto al trabajo de acompañamiento lector desarrollado en los ambientes presencial y virtual del modelo de aprendizaje $b$ learning.

ENCUESTA DE PERCEPCIÓN AMBIENTE VIRTUAL: conjunto de preguntas diseñadas para indagar sobre la percepción de los estudiantes frente al ambiente computacional implementado desde el curso en línea Comprendiendo Textos Expositivos ${ }^{2}$ y su per-

2 Alojado en $<$ www.readinglanguages.com/moodle $>$. 
tinencia pedagógica. La encuesta que se aplicó se trabajó desde la plataforma Moodle.

CuestionaRios y foros DE EVALUACIÓN EN ENTORNO VIRTUAL: estos instrumentos fueron configurados desde las herramientas y recursos de aprendizaje que ofrece la plataforma Moodle, orientados a medir cuantitativamente los niveles de comprensión lectora de acuerdo con los descriptores establecidos en las variables que guiaron la investigación.

\section{Descripción de la intervención en ambiente de aprendizaje b-learning}

Actualmente, las tecnologías de la información facilitan la configuración de otros ambientes de aprendizaje, uno de ellos es el b-learning, abreviatura de blended learning, concepto del inglés que en términos de enseñanza virtual se traduce como "formación combinada" o "enseñanza mixta" (Bartolomé, 2004). Se trata de una modalidad semipresencial de estudios que incluye formación no presencial (cursos on-line, conocidos genéricamente como e-learning) y formación presencial.

En el ambiente b-learning el formador desempeña el papel de orientador, de jalonador, pero usa en beneficio de la acción de enseñanza y aprendizaje el material didáctico que las herramientas Web y la Internet le proporcionan, para ejercer su labor en dos frentes: como tutor en línea (tutorías a distancia) y como educador presencial (cursos presenciales). La forma en que se combinen ambas estrategias depende de las necesidades específicas de ese curso, dotando así a la formación en línea de una gran flexibilidad y carácter pedagógico. No se trata solo de agregar tecnología a la clase, sino de reemplazar algunas actividades de aprendizaje por otras apoyadas con tecnología (Rosas, 2005).

El proceso de intervención se inicia con la aplicación de una encuesta metacognitiva sobre el manejo de hábitos lectores para textos expositivos, propuesta por López y Arciniegas (2004). Los resultados de esta aplicación mostraron algunas ausencias de conocimiento sobre las acciones a desarrollar en cada momento del proceso lector. Se dedicaron ocho semanas al proceso de experimentación. Este tiempo se compartió en dos entornos de trabajo: presencial y virtual, no siempre alternos, pues las actividades en cada uno fueron específicas e independientes. La manera de implementar el modelo se dio desde cinco principios básicos (Brown y Campione, 1986):

1. El profesor dirige el diálogo y modela las actividades de comprensión haciéndolas abiertas, explícitas y concretas. De esta forma los estudiantes tienen claro qué es lo que se requiere de ellos.

2. Las estrategias son siempre modeladas en contextos apropiados y no mediante la práctica de actividades aisladas y separadas. Es decir, no se trata de realizar actividades de resumen, de captación de ideas centrales, de vocabulario o de pensar que se avanza en la comprensión, desde la suma de estas actividades parciales, que se han trabajado de modo descontextualizado.

3. El diálogo y la discusión se centran tanto en el contenido del texto como en la comprensión de los estudiantes de la finalidad de las estrategias que se están utilizando.

4. El profesor da retroalimentación adaptada al nivel de comprensión de los estudiantes, animándolos a progresar gradualmente hacia una comprensión completa.

5. La responsabilidad de las actividades de comprensión se transfiere a los estudiantes tan pronto como es posible. Es decir, se trata de promover y provocar que ellos dirijan su propio aprendizaje a partir de los textos. 
Tabla 3. Componentes de la etapa de intervención

\begin{tabular}{|c|c|c|c|c|c|}
\hline \multirow[b]{2}{*}{ ACTIVIDAD } & \multicolumn{3}{|c|}{ ESTRATEGIAS } & \multicolumn{2}{|c|}{ RECURSOS B-LEARNING } \\
\hline & $\begin{array}{l}\text { Modelo lectura } \\
\text { focalizado }\end{array}$ & \multirow{6}{*}{ 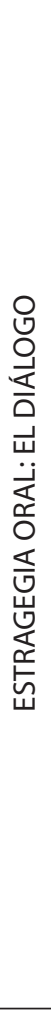 } & $\begin{array}{l}\text { Modelo lectura pano- } \\
\text { rámica }\end{array}$ & Ambiente presencial & Ambiente virtual \\
\hline Predicciones & $\begin{array}{l}\text { Activación de esque- } \\
\text { mas mentales (antes } \\
\text { de leer) }\end{array}$ & & $\begin{array}{l}\text { Activación de esque- } \\
\text { mas mentales } \\
\text { (antes de leer) }\end{array}$ & $\begin{array}{l}\text { Espacio de clase lide- } \\
\text { rado por el profesor } \\
\text { Utilización de la pre- } \\
\text { gunta para promover } \\
\text { la conversación oral }\end{array}$ & $\begin{array}{l}\text { One minute paper } \\
\text { (Ángelo y Cross, } \\
\text { 1993) } \\
\text { Traveling file } \\
\text { (Karre 1994) }\end{array}$ \\
\hline $\begin{array}{l}\text { Lectura guiada, } \\
\text { individual y en } \\
\text { pequeños } \\
\text { grupos }\end{array}$ & \multirow{2}{*}{$\begin{array}{l}\text { Estrategias de focali- } \\
\text { zación } \\
\text { (durante la lectura) } \\
\text { Estrategias de resolu- } \\
\text { ción de problemas } \\
\text { (durante la lectura) }\end{array}$} & & $\begin{array}{l}\text { Estrategias selectivas } \\
\text { (durante la lectura) }\end{array}$ & \multirow{3}{*}{$\begin{array}{l}\text { Actividad de modela- } \\
\text { miento del profesor } \\
\text { Trabajo independien- } \\
\text { te del estudiante } \\
\text { Actividades de con- } \\
\text { versación y trabajo en } \\
\text { pequeños grupos }\end{array}$} & \multirow{3}{*}{$\begin{array}{l}\text { Foro virtual } \\
\text { Talleres (trabajo } \\
\text { colaborativo) } \\
\text { Herramienta } \\
\text { Scorm } \\
\text { (3) Paquetes } \\
\text { MyUdutu }\end{array}$} \\
\hline Clarificación & & & $\begin{array}{l}\text { Estrategias de organi- } \\
\text { zación. } \\
\text { (durante la lectura) }\end{array}$ & & \\
\hline $\begin{array}{l}\text { Construcción } \\
\text { del significado } \\
\text { del texto }\end{array}$ & $\begin{array}{l}\text { Estrategias de resolu- } \\
\text { ción de problemas } \\
\text { (durante la lectura) }\end{array}$ & & $\begin{array}{l}\text { Estrategias de } \\
\text { elaboración } \\
\text { (durante la lectura) }\end{array}$ & & \\
\hline Autopreguntas & $\begin{array}{l}\text { Estrategias de elabo- } \\
\text { ración (después de la } \\
\text { lectura) }\end{array}$ & & $\begin{array}{l}\text { Estrategias de compro- } \\
\text { bación (después de la } \\
\text { lectura) }\end{array}$ & $\begin{array}{l}\text { Cuestionarios } \\
\text { Construcciones } \\
\text { escritas }\end{array}$ & $\begin{array}{l}\text { Actividad de eva- } \\
\text { luación combina- } \\
\text { da y en línea } \\
\text { Cuestionarios }\end{array}$ \\
\hline 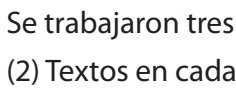 & 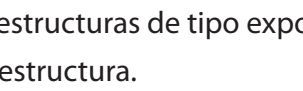 & & . & Tre & \\
\hline
\end{tabular}

Con respecto al entorno virtual, los estudiantes trabajaron en un curso on-line diseñado y habilitado en la plataforma Moodle. ${ }^{3}$ Este curso se implementó desde el manejo de objetos virtuales de aprendizaje. El curso virtual se estructuró en cuatro módulos, construidos desde la herramienta "MyUdutu" de Scorm. Los paquetes Scorm corresponden a bloques de material Web empaquetado de manera que se constituyen en objetos de aprendizaje y tienen un propósito pedagógico determinado.

La intervención pedagógica desde el ambiente virtual se desarrolló en diferentes momentos, es decir, algunas veces los estudiantes trabajaron por grupos en el aula de informática y otras, en espacios no académicos, generalmente fuera del colegio. La estructura didáctica del curso virtual es afín con el "método de enseñanza recíproca" para la comprensión lectora.

\section{Tratamiento de los datos}

Los resultados obtenidos durante la fase de experimentación fueron sometidos a un proceso de análisis desde la prueba estadística $\mathrm{T}$, manejada con el software Statics, versión 6.5. Los criterios empleados 
para el análisis estadístico se dieron en función de las hipótesis propuestas en la investigación. En el análisis de los resultados también se examinaron las frecuencias iniciales y finales, arrojadas en algunos de los ítems de la encuesta metacognitiva (López y Arciniegas, 2004).

\section{Resultados}

\section{Relación entre modelos de lectura y comprensión desde estilos cognitivos}

El análisis estadístico abordado en la investigación y el marco teórico que la sustenta permitieron mostrar que aunque no hay relaciones significativas entre los estilos cognitivos y los modelos de lectura, sí se hace evidente que las formas de procesamiento de la información se vuelven particulares, dependiendo del perfil cognitivo que caracteriza al sujeto lector. Los datos estadísticos muestran tendencias marcadas entre un modelo de lectura y un estilo cognitivo. En este caso, los estudiantes independientes de campo muestran un desempeño alto en los dos modelos: focalizado y panorámico.

Los estudiantes dependientes mantienen rangos más altos en el modelo focalizado sin generar diferencias significativas con los otros grupos en estudio. Los estudiantes dependientes de campo se perfilan con más bajos resultados en el modelo panorámico, pues, ya en la realización de las tareas que exige este tipo de lectura, algunas de ellas se abandonan o no se realizan y en otras hay poco margen de acierto. La tendencia al uso de una memoria a corto plazo (MCP) en función de almacenar información tiene una incidencia notoria en la lectura al restringir la realización de tareas cognitivas que demandan desechar información no relevante para establecer

Tabla 4. Medias obtenidas por los tres grupos de estilo cognitivo en modelo focalizado

\begin{tabular}{|c|c|c|c|}
\hline \multirow{2}{*}{$\begin{array}{l}\text { Nivel de comprensión } \\
\text { Estilo cognitivo }\end{array}$} & Dependientes & Intermedios & Independientes \\
\hline & $21,92308^{*}$ & $25,96154^{*}$ & $23,54167^{*}$ \\
\hline Local referencial & 18,65385 & 20,38462 & 21,45833 \\
\hline Global estructural & 10,57692 & $13,26923^{*}$ & $14,16667^{*}$ \\
\hline Intertextual & 51,15385 & 59,61538 & 59,16667 \\
\hline Total & 655,4615 & 855,7692 & 909,1667 \\
\hline Tiempo & & & \\
\hline
\end{tabular}

Tabla 5. Medias obtenidas por los tres grupos de estilo cognitivo en modelo panorámico

\begin{tabular}{|c|c|c|c|}
\hline \multirow{2}{*}{$\begin{array}{l}\text { Nivel de comprensión } \\
\text { Estilo cognitivo }\end{array}$} & Dependientes & Intermedios & Independientes \\
\hline Local referencial & 15,38462 & 16,53846 & 17,29167 \\
\hline Global estructural & $24,03846^{*}$ & $25,96154^{*}$ & $26,25000^{*}$ \\
\hline Intertextual & 7,88462 & 8,46154 & 9,79167 \\
\hline Total & 47,30769 & 50,96154 & 53,33333 \\
\hline Tiempo & 678,7308 & $495,1923^{*}$ & $524,7917^{*}$ \\
\hline
\end{tabular}

* Indica que el valor de $p$ marcó diferencias significativas. 
relaciones de niveles más complejos. Los estudiantes de estilo cognitivo intermedio, por su parte, se mantienen en un rango medio respecto de los dos anteriores, pero tienden a acercarse a los resultados de los independientes. Este grupo intermedio comparte rasgos de los dos polos, pero en este estudio el comportamiento del grupo se mostró cercano en resultados a los independientes de campo. En conclusión, los estudiantes de estilo cognitivo independiente alcanzan mejores resultados que los dependientes en las mismas condiciones y con iguales modelos de lectura y ambiente de aprendizaje. Un factor asociado a este resultado desde el modelo de enseñanza recíproca es el uso permanente de la conversación y el diálogo sobre las acciones adelantadas en cada etapa del proceso de lectura.

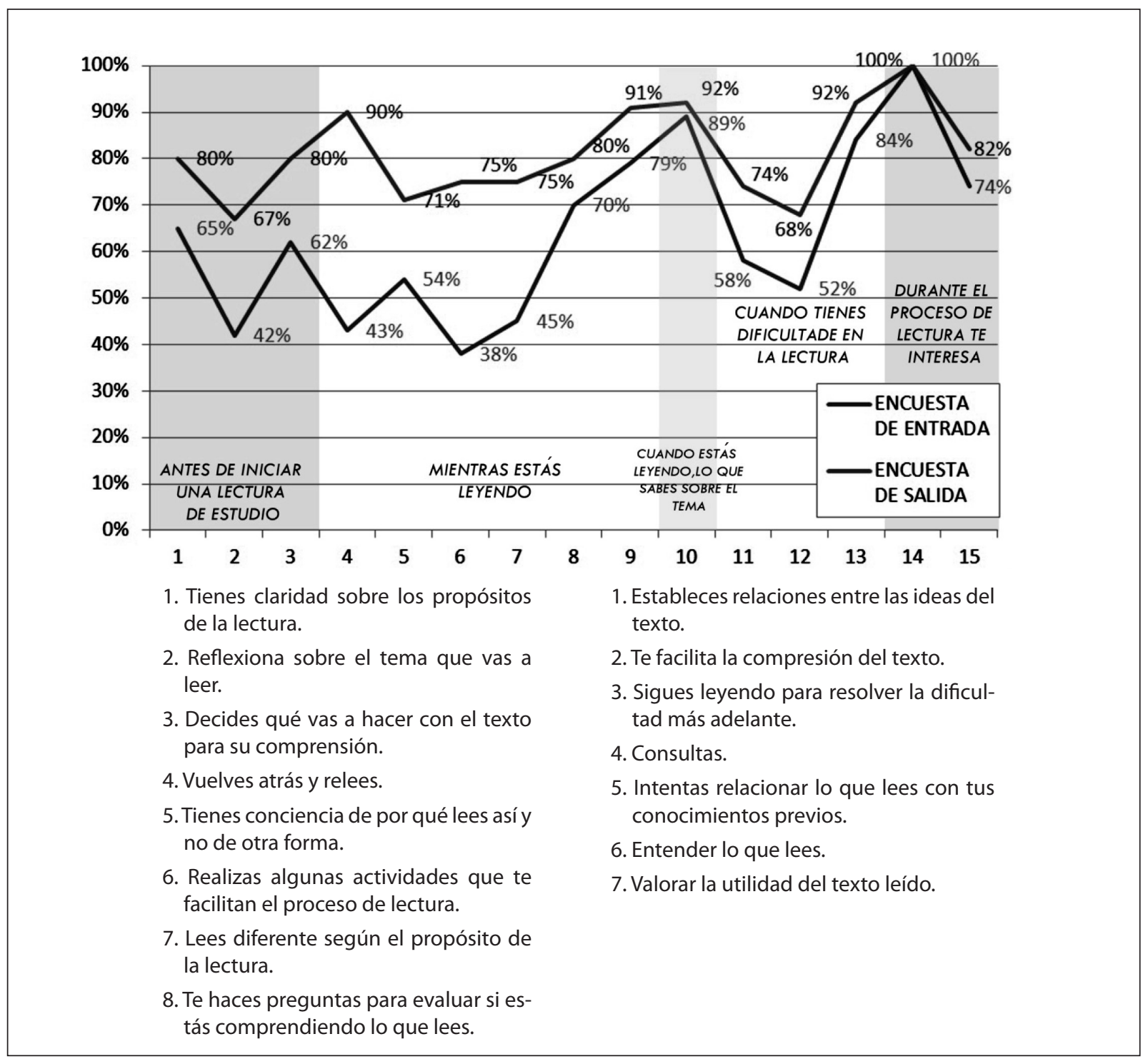

Figura 1. Respuestas correctas en Encuesta Metacognitiva pre y postest 


\section{Relaciones entre el modelo de enseñanza recíproca, la comprensión y la formación de actitudes metacognitivas}

En la siguiente figura se muestran los resultados obtenidos en la Encuesta Metacognitiva y contrasta los resultados de entrada (encuesta inicial) con los resultados de salida (encuesta final).

Los principios básicos que sustentan el modelo de enseñanza de la comprensión, denominado Modelo de Enseñanza Recíproca, se estructuran con el uso del diálogo y de la conversación, dirigida de manera colectiva o en pequeños grupos, como mecanismo para favorecer el modelamiento de estrategias entre pares. Estas condiciones no fueron verificadas estadísticamente de manera independiente al proceso de comprensión, sin embargo, desde los registros de observación directa y las reflexiones realizadas por los participantes del estudio se puede afirmar que inciden positivamente en el favorecimiento de la comprensión.

Las tareas de modelamiento de estrategias entre pares resultaron más efectivas y constantes en los grupos de estilo cognitivo independiente e intermedio, con menos éxito en el grupo de estilo cognitivo dependiente. Por otra parte, la actividad de mediación entre el texto, el lector y las estrategias de lectura orientada por el docente propició permanentes espacios de reflexión metacognitiva sobre el proceso lector de cada grupo de estudiantes. Por ello la función de la encuesta metacognitiva se desarrolló en dos direcciones: a) indagar el estado de metacognición que poseen los estudiantes al iniciar la experiencia y b) evaluar los avances obtenidos al finalizar la intervención. La figura 1 es una muestra de algunos de los ítems contenidos en cada una de las cinco partes que lo integran. Las opciones de res- puesta para la encuesta fueron "Sí" o "No". La figura 1 nos sitúa frente al nivel de respuestas afirmativas o correctas.

\section{Incidencia del ambiente de aprendizaje b-learning y la interacción verbal en la comprensión lectora}

Favorecer los procesos de enseñanza de la comprensión desde ambientes de aprendizaje y modelos de comprensión distintos a los tradicionales resulta altamente significativo para jóvenes que aún se sienten distantes de o bastante inexpertos en la lectura de tipo expositivo. El espacio de trabajo b-learning, estructurado en coherencia con el modelo de comprensión de la enseñanza recíproca permitió un mejor acompañamiento y monitoreo de la lectura de textos expositivos. Además, la implementación permanente del diálogo como estrategia oral favoreció espacios de trabajo colaborativo que para muchos estudiantes resultaron significativos, por cuanto sus propios procesos de aprendizaje pudieron incrementarse. De igual modo, esta experiencia pedagógica creó puentes de trabajo con otras disciplinas y fortaleció la idea de que "Leer para aprender es tarea de todos".

Resulta importante señalar que el éxito y la favorabilidad del ambiente b-learning permitieron el diseño y la implementación computacional de cuatro módulos de apoyo didáctico sobre Lectura Focalizada y Lectura Panorámica para trabajar, desde allí, la comprensión de textos expositivos. El estudio de las relaciones existentes entre estilos cognitivos y tipos de lectura se desarrolló de manera experimental vinculando dos escenarios de trabajo escolar: el escenario presencial (aula de clase) y el escenario virtual, curso e-learning "Comprendiendo textos expositivos". 


\section{Consideraciones finales}

El lenguaje de los libros de texto y las explicaciones del profesor son cada vez más formales y específicos. Los estudiantes deben comprender estos textos y dar cuenta de ello mediante otros similares. El uso de interacciones comunicativas orales vinculadas al proceso lector favorece significativamente la activación de esquemas mentales, el acercamiento al uso de estrategias cognitivas y metacognitivas y, por ende, la calidad del proceso de comprensión.

De manera general, los procesos de lectura no eficiente se caracterizan por que los lectores no participan activa ni estratégicamente en la lectura, no disponen de estrategias o no las emplean de manera adecuada, y se limitan, entonces, solo a la decodificación del texto. En consecuencia, es importante dedicarse a "llevar a cabo un trabajo sistemático y explícito de enseñanza de estrategias cognitivas y metacognitivas para la comprensión, que permita a los alumnos no solo involucrarse con los textos de manera adecuada a sus propósitos, sino que también les faciliten el control, la evaluación y la regulación de sus propios procesos durante su ejecución" (López y Arciniegas, 2004).

Dentro del modelo de enseñanza recíproca, orientado al fortalecimiento de la comprensión, el maestro orienta a los estudiantes mediante una serie de acciones sistemáticas, organizadas y con una meta clara. Esta función es ciertamente más relevante que en otros modelos y más exigente, pues requiere profundo conocimiento por parte del maestro y un adecuado modo de orientar el proceso de comprensión y aprendizaje a partir de la lectura de textos expositivos.

La influencia de la tecnologías en el mundo y, de manera particular, en los jóvenes constituye un aspecto favorable para su aprovechamiento pedagó- gico en la solución de problemáticas asociadas con la comprensión lectora y los modos particulares de aprender y de enseñar esta comprensión; a partir de ahí es posible incidir en los efectos que proyectan los estudiantes en diferentes campos de conocimiento mediados por la comprensión lectora de textos expositivos.

El trabajo con los grupos de estilo cognitivo desarrollado desde este estudio permitió hacer visible que el estilo cognitivo independiente se adapta más fácilmente al trabajo autónomo propuesto desde el aula virtual, pues varios de ellos señalan sentirse más cómodos avanzando a su propio ritmo, sin condicionarse por el nivel de trabajo de los demás, como sucede en algunos casos en el trabajo presencial. Por contraste, en el grupo de estilo cognitivo dependiente se hizo evidente la necesidad de estar en contacto constante con el maestro o con los pares para aclarar, reafirmar o recibir aprobación sobre el trabajo desarrollado en momentos parciales del proceso. En algunos casos los dependientes de campo manifestaron sentirse mucho mejor en el trabajo presencial, pues podían acceder a la ayuda y asesoría del maestro más fácilmente. Los estudiantes del grupo de estilo cognitivo intermedio se adaptan favorablemente al trabajo autónomo del aula virtual.

\section{Reconocimientos}

Este artículo se basa en la investigación "Relaciones entre modelos de lectura focalizada y panorámica y estilos cognitivos dependiente e independiente de campo, en ambientes de aprendizaje b-learning", desarrollada por la autora en la Escuela Normal Superior Distrital María Montessori. Hace parte de la línea de investigación "Comunidades de aprendizaje y ambientes virtuales", vinculada al grupo de investigación Kenta de la Universidad Pedagógica Nacional de Colombia. 


\section{Bibliografía}

Anderson, T. H. y Armbruster, B. B. (1984). Content Area Textbooks. En Learning to read in American Schools. Hillsdate, NJ: Lawrence Erlbaum.

Bartolomé, A. (2004). Blended Learning. Conceptos básicos. Pixel-Bit. Revista de Medios y Educación, 23: 7-20.

Brown, A. L. y Campione, J. C. (1986). Psychological Theory and the Study of learning Disabilities. American psychologist, 41(10).

Brown, A. L. y Palincasar, A. S. (1989). Guided, Cooperative Learning and Individual Knowledge Acquisition. En L. B. Resnick (ed.), Knowing, Learning and Instruction. Hillsdale, NJ: Lawrence Erlbaum.

Coll, C. (1988). Significado y sentido en el aprendizaje escolar. Infancia y Aprendizaje, 41. Madrid.

Gutiérrez, M. (1992). Comprensión y memoria de textos. Tratado de psicología general. J. Mayor y J. Pinillos (comps.), Memoria y representación (t. IV). Alhambra-Madrid.

Gutiérrez, S. (1997). Nuevos caminos en la lingüística. Aspectos de la competencia comunicativa. En J. Serrano y J. E. Martínez (coords), Didáctica de la lengua y literatura. Barcelona: OikosTau.

Jaimes, G. (2005). Competencias de la oralidad e inserción en la cultura escrita. Enunciación 10, 15-21. Bogotá, Fondo de publicaciones Universidad Distrital Francisco José de Caldas.

López, A. (1996). Tipología textual y técnicas de expresión oral. Lenguaje y textos, 9. Universidad de Murcia.
López, G. (1998). La lectura. Estrategias de comprensión de textos expositivos. Escuela de Ciencias del Lenguaje. Cali: Universidad del Valle.

López, G. y Arciniegas, E. (2004). Metacognición, lectura y construcción de conocimiento. El papel de los sujetos en el aprendizaje significativo. Cali: Universidad del Valle. Cátedra Unesco Meceal, Escuela de Ciencias del Lenguaje.

Lozano, A. (2005). Estilos de aprendizaje y enseñan$z a$. España: Editorial Mad.

Núñez, M. (2000). Enseñanza de la lengua oral: Una propuesta didáctica para el desarrollo de la competencia discursiva oral en la educación secundaria. Universidad de Granada.

Palincsar, A. S. y Brown, A. L. (1984). Reciprocal teaching of comprehension-fostering and comprehension-monitoring activities. Cognition and Instruction, 1, 117-175.

Richgels, D. et ál. (1990). Cómo enseñar la estructura del texto expositivo en la lectura y la escritura. En D. Muth (comp.). El texto expositivo. Estrategias para su comprensión. Argentina: Aique.

Rosales, C. (1987). Didáctica de la comunicación verbal. Madrid: Narcea.

Rosas, P. (2005). La gestión en ambientes virtuales de aprendizaje en los Posgrados de la U. de G. en tecnologías para internacionalizar el aprendizaje. Universidad de Guadalajara.

Sawa, H. (1966). Analytic thinking and synthetic thinking. Bulletin of Faculty of Education. Nagasaki University.

Solé, I. (1993). Estrategias de lectura y aprendizaje. Cuadernos de Pedagogía, 216, 25-27 
Solé, I. (2001). Estrategias de lectura. Barcelona: Grao.

Taboada, A. y Guthrie, J. (2006). La generación de preguntas y la comprensión lectora. Revista Latinoamericana de Lectura, 27(4).
Vigotsky, L. S. (1989). El desarrollo de los procesos sicológicos superiores. Barcelona: Grijalbo.

Vigotsky, L. S. (1978). Pensamiento y lenguaje. Buenos Aires: La Pleyade. 